\title{
Revisiting the NEH algorithm- the power of job insertion technique for optimizing the makespan in permutation flow shop scheduling
}

\author{
A. Baskar*
}

Principal, Apollo Engineering College, Mevaloorkuppam, Chennai-602 105, India

\begin{tabular}{l}
\hline C H R O N I C L E \\
\hline Article history: \\
Received July 212015 \\
Received in Revised Format \\
August 162015 \\
Accepted September 12015 \\
Available online \\
September 4 2015 \\
\hline Keywords: \\
NEH Algorithm \\
Job Insertion Technique \\
Flow Shop Scheduling: Makespan
\end{tabular}

\section{Introduction}

A flow shop scheduling problem (FSP) with $n$ jobs and $m$ machines has been a topic of research since 1950. Hejazi and Saghafian (2005) define the scheduling problem as "an effort to specify the order and timing of the processing of the jobs on machines, with an objective or objectives". Pinedo (2012) provides a vast literature about the scheduling theory, definitions, problems and tutorials. Among the many parameters that are being optimized, the makespan optimization is the choice of a good number of researchers in recent years. In permutation flow shop scheduling (PFSP), the total number of possibilities is $n$ !, where $n$ is the number of jobs to be scheduled. The problem is NP-Complete for more than 3 machines (Garey et al., 1976). In earlier days, Gantt charts (Gantt, 1919) were popular in scheduling which are still used by shop floor personnel for smaller problems. Many exact solutions have been proposed and analyzed by the researchers over the past decades. Most of them are based on branch and bound algorithm (example: Land \& Doig, 1960) or mathematical/ computer programming (example: Yin

* Corresponding author

E-mail: a.baaskar@gmail.com (A Baskar)

(c) 2016 Growing Science Ltd. All rights reserved. doi: 10.5267/j.jijiec.2015.9.001 
et al., 2010). The computation time grows exponentially with the size of the problem and hence, heuristics that yield reasonably accurate and acceptable results are more popular in this area.

Johnson's (1954) algorithm reported optimal makespan for two machines, $n$ number of jobs in a permutation flow shop problem. Since then, so many simple heuristics have been proposed over the years. The CDS algorithm proposed by Campbell et al. (1970) is the extension of Johnson's algorithm which selects the minimum makespan out of the $(m-1)$ enumerations. The $\mathrm{NEH}$ algorithm proposed by Nawaz et al. (1983) uses the powerful job insertion technique after arranging the jobs in the descending order of their total processing times. It selects the first two jobs as the initial partial sequence and other jobs are inserted one by one from the third job to obtain a final optimal makespan and its corresponding sequence. It has been generally agreed that the NEH algorithm is regarded as one of the best available simple, constructive heuristics even today. The complexity level and the quality of a few earlier simple heuristics were studied in detail by Taillard (1990) and it was concluded that the NEH algorithm is a better one for different sizes of problems varying from 9 to 50 jobs. Framinan et al. (2003) considered twenty two different approaches and eight different sorting criteria, totaling 176 approaches for every objective function. Additionally, for every objective function, the RANDOM choice of a sequence was considered. The processing times were drawn between 1 to 99 time units. It was concluded that for the makespan optimization, the NEH-insertion approach which selects the first two jobs as the initial partial initial sequence is a clear winner. A study conducted by Kalczynski and Kamburowski (2007) also reaffirms this conclusion.

The SPIRIT algorithm of Widmer and Hertz (1989) is also a constructive heuristic based on the wellknown traveling salesman problem. A few other constructive heuristic algorithms such as Rajendran (1993), Gajpal and Rajendran (2006) also use the powerful insertion technique of NEH to obtain the optimal sequence of jobs. Improvements have been performed on the NEH algorithm over the years to minimize the makespan as well as the computing time. The NEH heuristic algorithm itself has been an interesting area of research for many researchers. Many variants and improvements have been suggested over the last three decades. A few claims have been proved to be false also.

Sarin and Lefoka (1993) showed that their proposed algorithm was more effective when the number of machines is fewer than 100; but, NEH is better for larger number of machines. The performance of NEH algorithm was improved by improving the sorting step by Xiao-ping et al. (2004). Dong et al. (2008) ordered the jobs by the sums of the average processing time and the standard deviations of processing times to achieve the improvement. The Min-Max algorithm developed by Ronconi (2004) addresses the makespan minimization PFSP with no buffers. He found that the heuristic yields better results than the NEH algorithm for larger problems. Chakraborty and Laha (2007) modified the NEH algorithm using population-based technique and compared the results with NEH and HFC heuristic (Koulamas, 1998). They claimed that their algorithm outperforms NEH and HFC for the tested problem instances. The performance of the HFC algorithm is reported to outperform the NEH for non-permutation schedules.

Based on the concept of Johnson's algorithm, Kalczynski and Kamburowski (2008) proposed a new priority order combined with a simple tie-breaking method that leads to a heuristic that outperformed the NEH algorithm for all the problem sizes. Liu et al. (2012) proposed two new techniques to improve the $\mathrm{NEH}$ algorithm. The running time is reduced by introducing block properties and tie break rules to obtain solutions with smaller makespan. Singhal et al. (2012) considered the first four jobs from the sorted list and based on a parameter $k$, better partial sequences are considered for further job insertion. They claimed that the results are better than the NEH algorithm. However, the claim is not supported by rigorous validation except one numerical example and it is similar to the algorithm proposed by Chakraborty and Laha. Semančo and Modrák (2012) proposed one MOD approach for solving the PFSP. It uses the difference between the sums of processing times for each machine as a pair-splitting strategy to make two groups of the matrix of $n$-job and $m$-machine. Johnson's rule was used in the last step to get the minimum makespan. MOD's average relative percentage deviation for all the 120 Taillard's problems 
was reported as $6.88 \%$, which is inferior to that of the NEH. Ancău (2012) proposed two variants of heuristic algorithms to solve the permutation flow shop scheduling problem. The first algorithm is a constructive greedy heuristic which builds the optimal schedule of jobs on the basis of a selective-greedy process. The second algorithm is a modified version of the first algorithm, based on the iterative stochastic start.

Baskar and Xavior (2013) considered the first and last jobs, middle two jobs and the last two jobs as the initial partial sequences and analyzed the performance using Taillard (1993) instances. Initial sequencing of jobs is based on non-increasing order of their total processing times as proposed by NEH. FernandezViagas and Framinan (2014) presented a new tie-breaking mechanism based on an estimation of the idle times of the different subsequences in order to pick the one with the lowest value of the estimation. The computational experiments carried out by them showed that this mechanism outperforms the existing ones, both for the NEH and the iterated greedy algorithms for different CPU times.

Ribas and Mateo (2010) proposed two tools to improve the performance of NEH based heuristics. The first tool uses the reversibility property and the second one is a new tie-breaking strategy to use with the insertion phase of the NEH heuristic. They analyzed five initial solution procedures for both the problems. The algorithm recently proposed by Gupta and Chauhan (2015) performs better than many simple heuristics like Palmer, CDS and RA algorithms; but, could not perform better than the NEH algorithm. The solutions have been further improved by meta-heuristics. The results obtained from simple heuristics like NEH are mostly used as the candidate solution by these meta-heuristics to refine the solution further. Many meta-heuristics based on Simulated Annealing can be found in Osman and Potts (1989), Ogbu and Smith (1990) and Ishibuchi et al. (1995). Meta-heuristics based on Tabu Search techniques (Example: Nowicki \& Smutnicki, 1996) and Evolutionary algorithms (Example: Reeves, 1995; Caraffa et al., 2001; Onwubolu \& Davendra, 2006; Sayadi et al., 2010; Erdogmus, 2010) are also available in the literature.

\section{NEH Heuristic Algorithm}

NEH algorithm proposed by Nawaz et al. (1983) is widely accepted as one of the best simple heuristics for makespan minimization in permutation flow shop scheduling problems. The algorithm essentially consists of three steps. (i) All the jobs are scheduled in non-increasing order of their total processing times (ii) First two jobs are considered as an initial partial sequence (iii) Remaining jobs are inserted one by one starting from the third job at the place, which minimizes the partial makespan among the possible options. Total number of sequences to be enumerated is $[n(n+1) / 2-1]$ and hence, the complexity level is $\partial\left(n^{3} \mathrm{~m}\right)$. All the three components contribute to the efficient performance of the algorithm. Many initial sequences have been considered by other researchers in step (i) of NEH algorithm and the performances have been analyzed. (Example: Nagano \& Moccellin, 2002; Pour, 2001).

Ruiz and Maroto (2005) carried out an exhaustive review of eighteen constructive and improvement heuristics that include the Johnson (1954), Palmer (1965), CDS (1970), Gupta (1971), RA (1977) and NEH (1983) heuristics. They concluded that the NEH algorithm is one of the best simple algorithms available up to 2005 outperforming many meta-heuristics. For validating the algorithms, they have used Taillard (1993) benchmark problems. The reported average \% increase for the NEH algorithm over the best known solution for the 120 problem instances was $3.33 \%$. The Palmer, Gupta and Hundal and Rajgopal (1988) algorithms make use of a slope index assigned to each job. These heuristic algorithms sort the list of jobs using that weight as a sort key to create a feasible schedule.

\section{Proposed Variants}

Initial arrangement of the jobs may be based on any parameter and many researchers have tried in different ways to have a better initial sequencing. Also, the choice of initial partial sequence is numerous. 
The NEH algorithm selects the first two jobs after arranging them in non-increasing order of their total processing times. Out of the three steps in NEH algorithm, only the third step, that is, the job insertion technique is considered and the following variants listed in Table 1 are analyzed in this paper. Only two cases of initial arrangement of jobs are considered, (i) arranging them in non-increasing order of their total processing times and (ii) taking the data as it is without any sorting. The reason being, the purpose is to analyze the power of the job insertion technique. The initial sequence has been selected in many ways and the final sequence is constructed using the job insertion technique. Let,

$O S E Q$ - The sequence of the jobs in original data

$P S E Q$ - The sequence of the jobs after arranging them in non-increasing order of total processing times $n-$ Number of jobs

$m$ - Number of machines

\section{Table 1}

Variants of NEH algorithm

\begin{tabular}{|c|c|c|c|}
\hline S.No & Algorithm & Initial Sequence & Further Job Insertion \\
\hline 1 & $\mathrm{NEH}$ & First two jobs from PSEQ & Other jobs one by one from PSEQ \\
\hline 2 & NEHAB1 & $\begin{array}{l}\text { Job nos. }[7,14] \text { if } n=20 ;[17,34] \text { if } n=50 ;[34,68] \text { if } \\
n=100 ;[67,134] \text { if } n=200 \text { and }[167,334] \text { if } n=500 \text { from } \\
\text { OSEQ }\end{array}$ & Other jobs one by one from PSEQ \\
\hline 3 & NEHAB2 & $\begin{array}{l}\text { Job nos. }[1,11] \text { if } n=20 ;[1,26] \text { if } n=50 ;[1,51] \text { if } n=100 \text {; } \\
{[1,101] \text { if } n=200 \text { and }[1,251] \text { if } n=500 \text { from OSEQ }}\end{array}$ & Other jobs one by one from PSEQ \\
\hline 4 & NEHAB3 & First two jobs from OSEQ & $\begin{array}{l}\text { Other jobs one by one from } \\
\text { OSEQ }\end{array}$ \\
\hline 5 & NEHAB4 & $\begin{array}{l}\text { Job nos. }[1,11] \text { if } n=20 ;[1,26] \text { if } n=50 ;[1,51] \text { if } n=100 \text {; } \\
{[1,101] \text { if } n=200 \text { and }[1,251] \text { if } n=500 \text { from PSEQ }}\end{array}$ & Other jobs one by one from PSEQ \\
\hline 6 & NEHAB5 & $\begin{array}{l}\text { Job nos. }[10,11] \text { if } n=20 ;[25,26] \text { if } n=50 ;[50,51] \text { if } \\
n=100 ;[100,101] \text { if } n=200 \text { and }[250,251] \text { if } n=500 \text { from } \\
\text { OSEQ }\end{array}$ & Other jobs one by one from PSEQ \\
\hline 7 & NEHAB6 & $\begin{array}{l}\text { Job nos. }[7,14] \text { if } n=20 ;[17,34] \text { if } n=50 ;[34,68] \text { if } \\
n=100 ;[67,134] \text { if } n=200 \text { and }[167,334] \text { if } n=500 \text { from } \\
\text { PSEQ }\end{array}$ & Other jobs one by one from PSEQ \\
\hline 8 & NEHAB7 & $\begin{array}{l}\text { Job nos. }[7,14] \text { if } n=20 ;[17,34] \text { if } n=50 ;[34,68] \text { if } \\
n=100 ;[67,134] \text { if } n=200 \text { and }[167,334] \text { if } n=500 \text { from } \\
\text { OSEQ }\end{array}$ & $\begin{array}{l}\text { Other jobs one by one from } \\
\text { OSEQ }\end{array}$ \\
\hline
\end{tabular}

\section{Results and Discussion}

The algorithms are coded in MATLAB R2008a and run in an i5 PC with 4 GB RAM. For validating the algorithms, the 120 numbers of Taillard (1993) benchmark instances have been used. They are grouped into 12 sets of 10 problems each with varying sizes having 20, 50, 100, 200 and 500 jobs and 5, 10 and 20 machines. The lower bounds are available in the Taillard paper itself and the known upper bounds as of April 2005 (www.mistic.heig-vd.ch/taillard/problems.dir/ordonnancement,dir/flowshop.dir/best_lb_up.txt) were taken from the website. The obtained makespan are reproduced in Table 2. The performance measure being considered for the analysis purpose is:

\% increase in Makespan from the known upper bound (Relative Deviation, RD), $=$ (Makespan obtained- Known Upper Bound) x 100) / Known Upper Bound.

The average \% increase in Makespan from the known upper bound (Relative Deviation, RD), are computed and presented Table 3. 
Table 2

Makespan for different Algorithms using Taillard Problems

\begin{tabular}{|c|c|c|c|c|c|c|c|c|c|}
\hline $\mathrm{m} / \mathrm{c} \times \mathrm{Job}$ & UB & $\mathrm{NEH}$ & NEHAB1 & NEHAB2 & NEHAB3 & NEHAB4 & NEHAB5 & NEHAB6 & NEHAB7 \\
\hline & 1278 & 1286 & 1286 & 1299 & 1310 & 1299 & 1300 & 1297 & 1297 \\
\hline & 1359 & 1365 & 1367 & 1365 & 1383 & 1383 & 1377 & 1383 & 1383 \\
\hline & 1081 & 1159 & 1139 & 1140 & 1132 & 1150 & 1123 & 1139 & 1141 \\
\hline & 1293 & 1325 & 1337 & 1314 & 1355 & 1364 & 1314 & 1315 & 1354 \\
\hline \multirow[t]{10}{*}{$5 \times 20$} & 1235 & 1305 & 1244 & 1250 & 1277 & 1244 & 1305 & 1305 & 1283 \\
\hline & 1195 & 1228 & 1212 & 1229 & 1224 & 1231 & 1234 & 1236 & 1237 \\
\hline & 1234 & 1278 & 1280 & 1251 & 1276 & 1269 & 1266 & 1251 & 1253 \\
\hline & 1206 & 1223 & 1224 & 1278 & 1248 & 1223 & 1221 & 1257 & 1239 \\
\hline & 1230 & 1291 & 1270 & 1291 & 1263 & 1278 & 1253 & 1245 & 1258 \\
\hline & 1108 & 1151 & 1153 & 1151 & 1131 & 1151 & 1161 & 1153 & 1153 \\
\hline & 1582 & 1680 & 1655 & 1631 & 1665 & 1680 & 1631 & 1623 & 1658 \\
\hline & 1659 & 1729 & 1729 & 1735 & 1753 & 1776 & 1729 & 1729 & 1767 \\
\hline & 1496 & 1557 & 1541 & 1550 & 1602 & 1566 & 1574 & 1570 & 1583 \\
\hline & 1377 & 1439 & 1439 & 1441 & 1465 & 1424 & 1443 & 1452 & 1437 \\
\hline \multirow[t]{10}{*}{$10 \times 20$} & 1419 & 1502 & 1515 & 1493 & 1503 & 1502 & 1505 & 1498 & 1511 \\
\hline & 1397 & 1453 & 1473 & 1447 & 1515 & 1434 & 1487 & 1474 & 1520 \\
\hline & 1484 & 1562 & 1550 & 1581 & 1529 & 1527 & 1541 & 1523 & 1557 \\
\hline & 1538 & 1609 & 1585 & 1663 & 1635 & 1605 & 1599 & 1583 & 1619 \\
\hline & 1593 & 1647 & 1644 & 1652 & 1645 & 1647 & 1646 & 1678 & 1668 \\
\hline & 1591 & 1653 & 1683 & 1639 & 1670 & 1634 & 1639 & 1635 & 1662 \\
\hline & 2297 & 2410 & 2397 & 2422 & 2479 & 2402 & 2402 & 2394 & 2446 \\
\hline & 2099 & 2150 & 2160 & 2126 & 2167 & 2184 & 2159 & 2163 & 2175 \\
\hline & 2326 & 2411 & 2382 & 2407 & 2512 & 2405 & 2403 & 2398 & 2449 \\
\hline & 2223 & 2262 & 2281 & 2240 & 2278 & 2280 & 2240 & 2264 & 2283 \\
\hline \multirow[t]{10}{*}{$20 \times 20$} & 2291 & 2397 & 2397 & 2367 & 2341 & 2367 & 2363 & 2383 & 2435 \\
\hline & 2226 & 2349 & 2281 & 2337 & 2354 & 2328 & 2357 & 2343 & 2279 \\
\hline & 2273 & 2362 & 2348 & 2356 & 2386 & 2338 & 2356 & 2393 & 2350 \\
\hline & 2200 & 2249 & 2249 & 2257 & 2314 & 2273 & 2265 & 2282 & 2283 \\
\hline & 2237 & 2320 & 2299 & 2320 & 2387 & 2330 & 2320 & 2349 & 2384 \\
\hline & 2178 & 2277 & 2235 & 2296 & 2289 & 2277 & 2276 & 2300 & 2297 \\
\hline & 2724 & 2733 & 2733 & 2733 & 2729 & 2733 & 2733 & 2733 & 2744 \\
\hline & 2834 & 2843 & 2882 & 2843 & 2933 & 2843 & 2843 & 2882 & 2925 \\
\hline & 2621 & 2640 & 2630 & 2638 & 2709 & 2640 & 2647 & 2630 & 2676 \\
\hline & 2751 & 2782 & 2782 & 2832 & 2778 & 2804 & 2812 & 2811 & 2805 \\
\hline \multirow[t]{10}{*}{$5 \times 50$} & 2863 & 2868 & 2868 & 2868 & 2891 & 2868 & 2868 & 2897 & 2891 \\
\hline & 2829 & 2850 & 2840 & 2863 & 2873 & 2841 & 2850 & 2841 & 2882 \\
\hline & 2725 & 2758 & 2745 & 2763 & 2751 & 2753 & 2753 & 2767 & 2736 \\
\hline & 2683 & 2721 & 2698 & 2688 & 2725 & 2707 & 2710 & 2713 & 2739 \\
\hline & 2552 & 2576 & 2575 & 2574 & 2613 & 2574 & 2574 & 2574 & 2596 \\
\hline & 2782 & 2790 & 2786 & 2793 & 2794 & 2789 & 2786 & 2798 & 2791 \\
\hline & 2991 & 3135 & 3151 & 3156 & 3194 & 3176 & 3178 & 3117 & 3140 \\
\hline & 2867 & 3032 & 3012 & 3038 & 3046 & 3024 & 3013 & 3097 & 3028 \\
\hline & 2839 & 2986 & 2987 & 2987 & 3077 & 2973 & 2995 & 3009 & 3156 \\
\hline & 3063 & 3198 & 3136 & 3139 & 3202 & 3177 & 3147 & 3203 & 3218 \\
\hline \multirow[t]{10}{*}{$10 \times 50$} & 2976 & 3160 & 3097 & 3129 & 3142 & 3153 & 3144 & 3174 & 3149 \\
\hline & 3006 & 3178 & 3184 & 3172 & 3154 & 3165 & 3165 & 3177 & 3139 \\
\hline & 3093 & 3277 & 3291 & 3289 & 3239 & 3277 & 3310 & 3265 & 3296 \\
\hline & 3037 & 3123 & 3196 & 3185 & 3170 & 3153 & 3168 & 3171 & 3167 \\
\hline & 2897 & 3002 & 3062 & 3078 & 3062 & 3079 & 3035 & 3029 & 3032 \\
\hline & 3065 & 3257 & 3229 & 3276 & 3263 & 3209 & 3294 & 3232 & 3285 \\
\hline & 3850 & 4082 & 4078 & 4044 & 4099 & 4044 & 4069 & 4122 & 4078 \\
\hline & 3704 & 3921 & 4009 & 4011 & 4029 & 4021 & 3869 & 3865 & 4047 \\
\hline & 3640 & 3927 & 3882 & 3890 & 3882 & 3852 & 3894 & 3887 & 3907 \\
\hline & 3723 & 3969 & 3936 & 3942 & 4037 & 3934 & 3991 & 3966 & 3991 \\
\hline \multirow[t]{6}{*}{$20 \times 50$} & 3611 & 3835 & 3879 & 3860 & 3878 & 3865 & 3899 & 3861 & 3859 \\
\hline & 3681 & 3914 & 3901 & 3892 & 3968 & 3902 & 3917 & 3903 & 3996 \\
\hline & 3704 & 3952 & 3897 & 3958 & 3986 & 3949 & 3936 & 3952 & 3939 \\
\hline & 3691 & 3938 & 3925 & 3903 & 3976 & 3932 & 3950 & 3926 & 3982 \\
\hline & 3743 & 3952 & 3922 & 3970 & 3972 & 3997 & 3996 & 4004 & 3971 \\
\hline & 3756 & 4079 & 3991 & 3953 & 3958 & 3991 & 4069 & 4028 & 3964 \\
\hline
\end{tabular}


Table 2

Makespan for different Algorithms using Taillard Problems (Contd...)

\begin{tabular}{|c|c|c|c|c|c|c|c|c|c|}
\hline $\mathrm{m} / \mathrm{c} \times \mathrm{Job}$ & UB & $\mathrm{NEH}$ & NEHAB1 & NEHAB2 & NEHAB3 & NEHAB4 & NEHAB5 & NEHAB6 & NEHAB7 \\
\hline & 5493 & 5519 & 5519 & 5582 & 5527 & 5560 & 5552 & 5552 & 5533 \\
\hline & 5268 & 5348 & 5348 & 5341 & 5326 & 5348 & 5348 & 5329 & 5350 \\
\hline & 5175 & 5219 & 5219 & 5220 & 5262 & 5253 & 5208 & 5201 & 5247 \\
\hline & 5014 & 5023 & 5023 & 5021 & 5028 & 5023 & 5049 & 5023 & 5022 \\
\hline & 5250 & 5266 & 5266 & 5261 & 5298 & 5261 & 5267 & 5267 & 5263 \\
\hline \multirow[t]{9}{*}{$5 \times 100$} & 5135 & 5139 & 5141 & 5139 & 5192 & 5139 & 5139 & 5139 & 5192 \\
\hline & 5246 & 5259 & 5259 & 5266 & 5340 & 5289 & 5292 & 5270 & 5304 \\
\hline & 5094 & 5120 & 5108 & 5113 & 5137 & 5105 & 5131 & 5126 & 5151 \\
\hline & 5448 & 5489 & 5489 & 5489 & 5514 & 5489 & 5500 & 5489 & 5504 \\
\hline & 5322 & 5341 & 5349 & 5349 & 5357 & 5342 & 5346 & 5346 & 5382 \\
\hline & 5770 & 5846 & 5881 & 5866 & 5920 & 5864 & 5893 & 5894 & 5882 \\
\hline & 5349 & 5453 & 5421 & 5478 & 5550 & 5431 & 5466 & 5431 & 5533 \\
\hline & 5676 & 5824 & 5752 & 5756 & 5841 & 5775 & 5756 & 5834 & 5810 \\
\hline & 5781 & 5929 & 5986 & 5982 & 6112 & 5958 & 5988 & 5929 & 6086 \\
\hline \multirow[t]{10}{*}{$10 \times 100$} & 5467 & 5679 & 5621 & 5639 & 5672 & 5717 & 5693 & 5687 & 5628 \\
\hline & 5303 & 5375 & 5365 & 5366 & 5447 & 5347 & 5376 & 5369 & 5468 \\
\hline & 5595 & 5704 & 5718 & 5719 & 5712 & 5713 & 5744 & 5678 & 5739 \\
\hline & 5617 & 5760 & 5740 & 5761 & 5829 & 5789 & 5762 & 5729 & 5808 \\
\hline & 5871 & 6032 & 6013 & 6040 & 6037 & 6018 & 6014 & 6009 & 6063 \\
\hline & 5845 & 5918 & 5903 & 5903 & 5925 & 5920 & 5909 & 5928 & 6041 \\
\hline & 6202 & 6541 & 6582 & 6574 & 6631 & 6586 & 6505 & 6603 & 6581 \\
\hline & 6183 & 6523 & 6524 & 6494 & 6502 & 6516 & 6571 & 6622 & 6534 \\
\hline & 6271 & 6639 & 6628 & 6632 & 6583 & 6656 & 6664 & 6603 & 6609 \\
\hline & 6269 & 6557 & 6590 & 6624 & 6687 & 6572 & 6609 & 6608 & 6572 \\
\hline \multirow[t]{10}{*}{$20 \times 100$} & 6314 & 6695 & 6645 & 6721 & 6720 & 6640 & 6612 & 6658 & 6692 \\
\hline & 6364 & 6664 & 6741 & 6670 & 6772 & 6696 & 6741 & 6742 & 6716 \\
\hline & 6268 & 6632 & 6567 & 6657 & 6605 & 6628 & 6623 & 6564 & 6657 \\
\hline & 6401 & 6739 & 6774 & 6744 & 6869 & 6778 & 6791 & 6723 & 6846 \\
\hline & 6275 & 6677 & 6563 & 6655 & 6636 & 6560 & 6614 & 6617 & 6737 \\
\hline & 6434 & 6677 & 6762 & 6710 & 6741 & 6771 & 6727 & 6734 & 6785 \\
\hline & 10862 & 10912 & 10942 & 10942 & 11033 & 10952 & 11032 & 10954 & 10988 \\
\hline & 10480 & 10716 & 10735 & 10722 & 10728 & 10660 & 10640 & 10702 & 10770 \\
\hline & 10922 & 11025 & 11028 & 11048 & 11172 & 11062 & 11098 & 11027 & 11175 \\
\hline & 10889 & 11057 & 11057 & 11057 & 11057 & 11057 & 11057 & 11057 & 10954 \\
\hline \multirow[t]{10}{*}{$10 \times 200$} & 10524 & 10645 & 10623 & 10617 & 10689 & 10636 & 10658 & 10654 & 10693 \\
\hline & 10329 & 10458 & 10458 & 10460 & 10559 & 10530 & 10439 & 11449 & 10626 \\
\hline & 10854 & 10989 & 10991 & 11039 & 11053 & 10967 & 10991 & 11005 & 11003 \\
\hline & 10730 & 10829 & 10856 & 10887 & 10913 & 10851 & 10867 & 10846 & 10980 \\
\hline & 10438 & 10574 & 10625 & 10574 & 10639 & 10578 & 10580 & 10635 & 10599 \\
\hline & 10675 & 10807 & 10870 & 10811 & 10946 & 10773 & 10822 & 10823 & 10927 \\
\hline & 11195 & 11625 & 11663 & 11600 & 11734 & 11648 & 11668 & 11597 & 11630 \\
\hline & 11203 & 11675 & 11798 & 11741 & 11815 & 11710 & 11771 & 11736 & 11847 \\
\hline & 11281 & 11852 & 11858 & 11806 & 11848 & 11766 & 11810 & 11848 & 11831 \\
\hline & 11275 & 11803 & 11729 & 11728 & 11767 & 11709 & 11780 & 11800 & 11892 \\
\hline \multirow[t]{10}{*}{$20 \times 200$} & 11259 & 11685 & 11691 & 11719 & 11763 & 11685 & 11692 & 11649 & 11803 \\
\hline & 11176 & 11629 & 11679 & 11657 & 11699 & 11759 & 11725 & 11661 & 11709 \\
\hline & 11360 & 11833 & 11803 & 11758 & 11878 & 11796 & 11852 & 11815 & 11942 \\
\hline & 11334 & 11913 & 11797 & 11783 & 11844 & 11896 & 11851 & 11870 & 11847 \\
\hline & 11192 & 11673 & 11646 & 11619 & 11767 & 11688 & 11651 & 11625 & 11733 \\
\hline & 11288 & 11869 & 11820 & 11754 & 11875 & 11784 & 11703 & 11755 & 11854 \\
\hline & 26059 & 26670 & 26703 & 26686 & 26802 & 26731 & 26843 & 26733 & 26843 \\
\hline & 26520 & 27232 & 27238 & 27258 & 27255 & 27144 & 27276 & 27312 & 27292 \\
\hline & 26371 & 26848 & 26917 & 26887 & 27108 & 26982 & 26861 & 26977 & 27182 \\
\hline & 26456 & 27055 & 26993 & 26913 & 27043 & 26946 & 26943 & 26934 & 26990 \\
\hline \multirow[t]{6}{*}{$20 \times 500$} & 26334 & 26727 & 26784 & 26887 & 26953 & 26813 & 26957 & 26830 & 26900 \\
\hline & 26477 & 26992 & 26941 & 27157 & 27079 & 27083 & 27030 & 27074 & 27104 \\
\hline & 26389 & 26797 & 26746 & 26702 & 26932 & 26838 & 26834 & 26783 & 27050 \\
\hline & 26560 & 27138 & 27237 & 27073 & 27153 & 27153 & 27165 & 27205 & 27270 \\
\hline & 26005 & 26631 & 26618 & 26652 & 26924 & 26584 & 26586 & 26602 & 26734 \\
\hline & 26457 & 26984 & 26955 & 26977 & 27044 & 27080 & 26932 & 27021 & 27080 \\
\hline
\end{tabular}


Table 3

Average \% increase in Makespan from the known upper bound (Relative Deviation)

\begin{tabular}{ccccccccc}
\hline Size & NEH (Ref.) & NEHAB1 & NEHAB2 & NEHAB3 & NEHAB4 & NEHAB5 & NEHAB6 & NEHAB7 \\
\hline $5 \times 20$ & 3.300288 & 2.466798 & 2.94145 & 3.12553 & 3.105381 & 2.797727 & 3.0305926 & 3.153593 \\
$10 \times 20$ & 4.601116 & 4.503733 & 4.611437 & 5.632414 & 4.318363 & 4.397786 & 4.1938635 & 5.613124 \\
$20 \times 20$ & 3.730891 & 3.028943 & 3.464978 & 5.152144 & 3.73614 & 3.534852 & 4.109836 & 4.595636 \\
$5 \times 50$ & 0.727204 & 0.639589 & 0.845576 & 1.589029 & 0.693401 & 0.7814 & 1.0256895 & 1.539656 \\
$10 \times 50$ & 5.072897 & 5.067393 & 5.418086 & 5.770844 & 5.208919 & 5.408071 & 5.511376 & 5.974275 \\
$20 \times 50$ & 6.648051 & 6.251361 & 6.261627 & 7.234613 & 6.431331 & 6.707959 & 6.4955621 & 7.099907 \\
$5 \times 100$ & 0.527212 & 0.522582 & 0.631669 & 1.020972 & 0.687648 & 0.733877 & 0.5604858 & 0.957314 \\
$10 \times 100$ & 2.21498 & 1.994005 & 2.194672 & 3.151677 & 2.23288 & 2.359485 & 2.1542496 & 3.16718 \\
$20 \times 100$ & 5.344636 & 5.390678 & 5.561187 & 5.977305 & 5.434273 & 5.520767 & 5.5521205 & 5.95049 \\
$10 \times 200$ & 1.230268 & 1.392741 & 1.363868 & 1.957031 & 1.280906 & 1.386078 & 2.3294067 & 1.892757 \\
$20 \times 200$ & 4.435269 & 4.37247 & 4.088959 & 4.822075 & 4.334303 & 4.389163 & 4.2574951 & 4.908013 \\
$20 \times 500$ & 2.066128 & 2.088155 & 2.11122 & 2.530346 & 2.17239 & 2.200622 & 2.2164883 & 2.586686 \\
\hline Mean & 3.324912 & 3.143204 & 3.291227 & 3.996998 & 3.302995 & 3.351482 & 3.4530972 & 3.953219 \\
\hline Rank & 4 & 1 & 2 & 8 & 3 & 5 & 6 & 7 \\
\hline
\end{tabular}

In case of the NEH Algorithm, Ruiz reported an average increase of $3.33 \%$ over the best known solution for the 120 problem instances. Whereas, the average obtained by the author is $3.3249 \%$. The CDS, Palmer, Gupta and RA heuristics are not considered as the deviations are very high when compared to the other heuristics. The average increase is below $4 \%$ of all the cases analyzed. Even the NEHAB3 algorithm that selects the first two jobs as the initial partial sequence from the original data without sequencing the jobs initially, reports a deviation less than $4 \%$. Also, the algorithm NEHAB7 that selects different jobs initially from the original data reports a deviation of $3.953219 \%$, which is slightly better than NEHAB3. Only, the job insertion technique is used to construct the final schedule from the original data. The performances of these two are better than most of the simple heuristics, including that of Suliman (2000) in which the reported increase was $6.21 \%$.

The heuristics analyzed in this paper perform better than a few meta-heuristics also:

GA Sprit - $7.15 \%$ (Widmer \& Hertz, 1989);

One GA - $4.75 \%$ (Chen et al., 1995);

One Hybrid GA - $8.92 \%$ (Murata et al., 1996) and

Another GA - 12.53 \% (Ponnambalam et al., 2001).

The individual performances are also analyzed and reported in Table 4 with respect to the number of problem instances they report better makespan when compared with the NEH (reference). NEHAB1 reports better results in case of 57 instances, whereas, $\mathrm{NEH}$ reports in 45 problems only. In 18 cases, the reported makespan are the same. NEHAB1 reports a mean deviation of $3.143204 \%$, which is the least among the heuristics including the NEH.

\section{Table 4}

Individual Performance based on the Number of Instances

\begin{tabular}{|c|c|c|c|c|c|}
\hline S.No. & Algorithm & Self & NEH (Ref.) & Same Makespan & Rank \\
\hline 1 & NEHAB1 & 57 & 45 & 18 & 1 \\
\hline 2 & NEHAB2 & 49 & 59 & 12 & 5 \\
\hline 3 & NEHAB3 & 26 & 93 & 01 & 8 \\
\hline 4 & NEHAB4 & 53 & 50 & 17 & 2 \\
\hline 5 & NEHAB5 & 47 & 63 & 10 & 6 \\
\hline 6 & NEHAB6 & 52 & 59 & 09 & 4 \\
\hline 7 & NEHAB7 & 28 & 92 & 00 & 7 \\
\hline
\end{tabular}

The rank of NEH is taken as 3 in Table 4. This ranking is different from the one based on the mean deviation from the known upper bounds. NEH holds the position of 4 there in Table 3 . In both the cases, NEHAB1 that selects the initial partial sequence as the job numbers $[7,14]$ if $n=20$; $[17,34]$ if $n=50$; $[34,68]$ if $n=100 ;[67,134]$ if $n=200$ and $[167,334]$ if $n=500$ from the original data performs extremely 
better. Other jobs are inserted one by one from the sequence of the jobs after arranging them in nonincreasing order of total processing times. The mean deviations are presented graphically in Fig. 1 to Fig. 3.

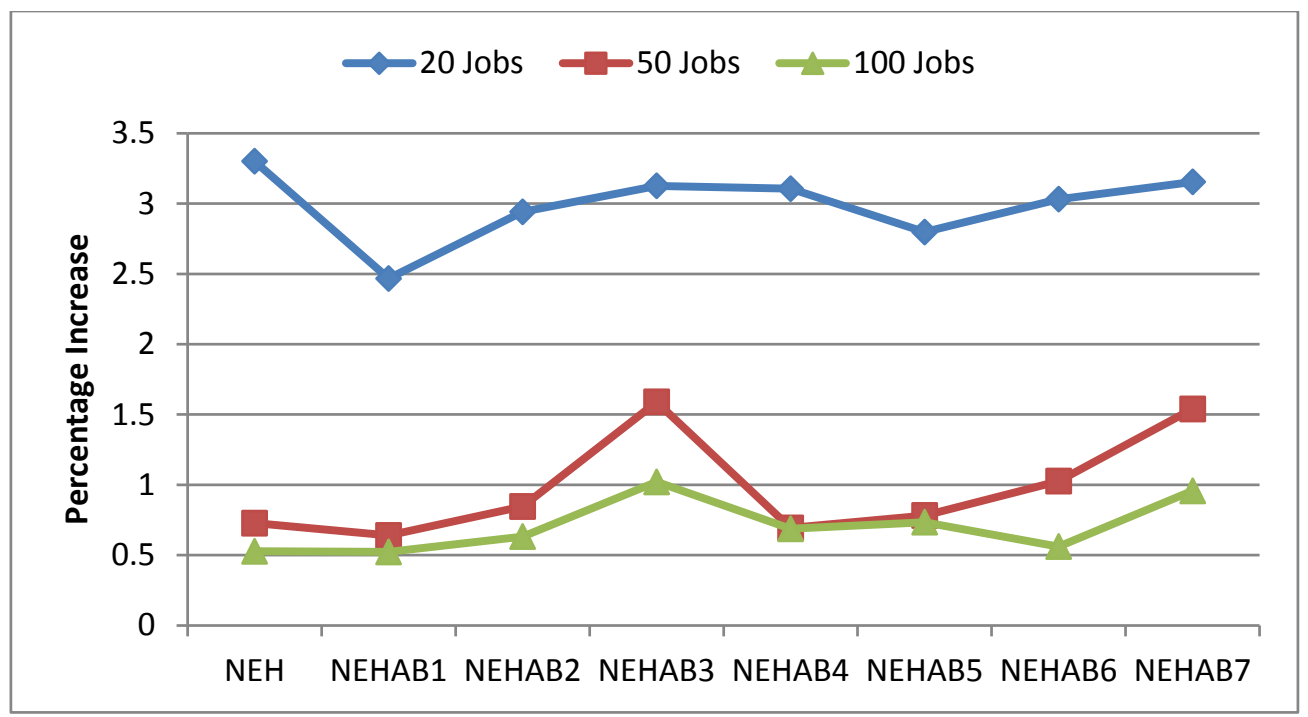

Fig. 1. Percent Increase Plot for 5 Machines

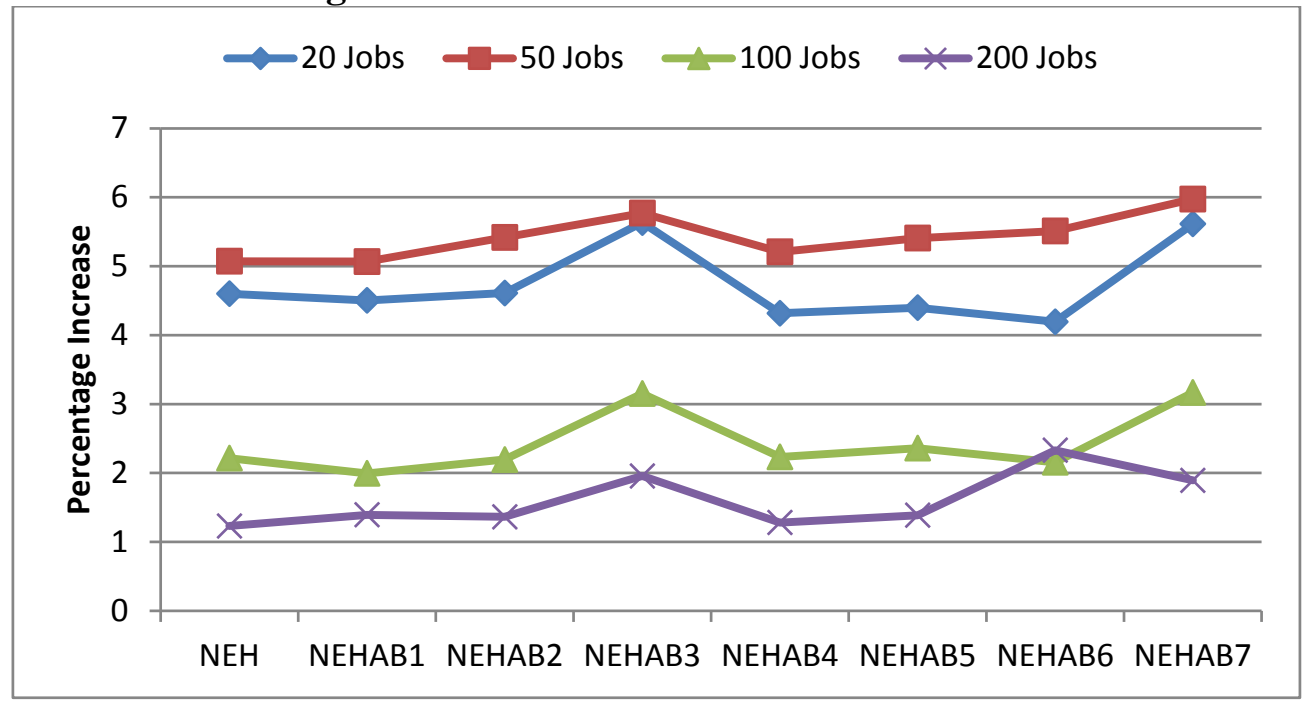

Fig. 2. Percent Increase Plot for 10 Machines

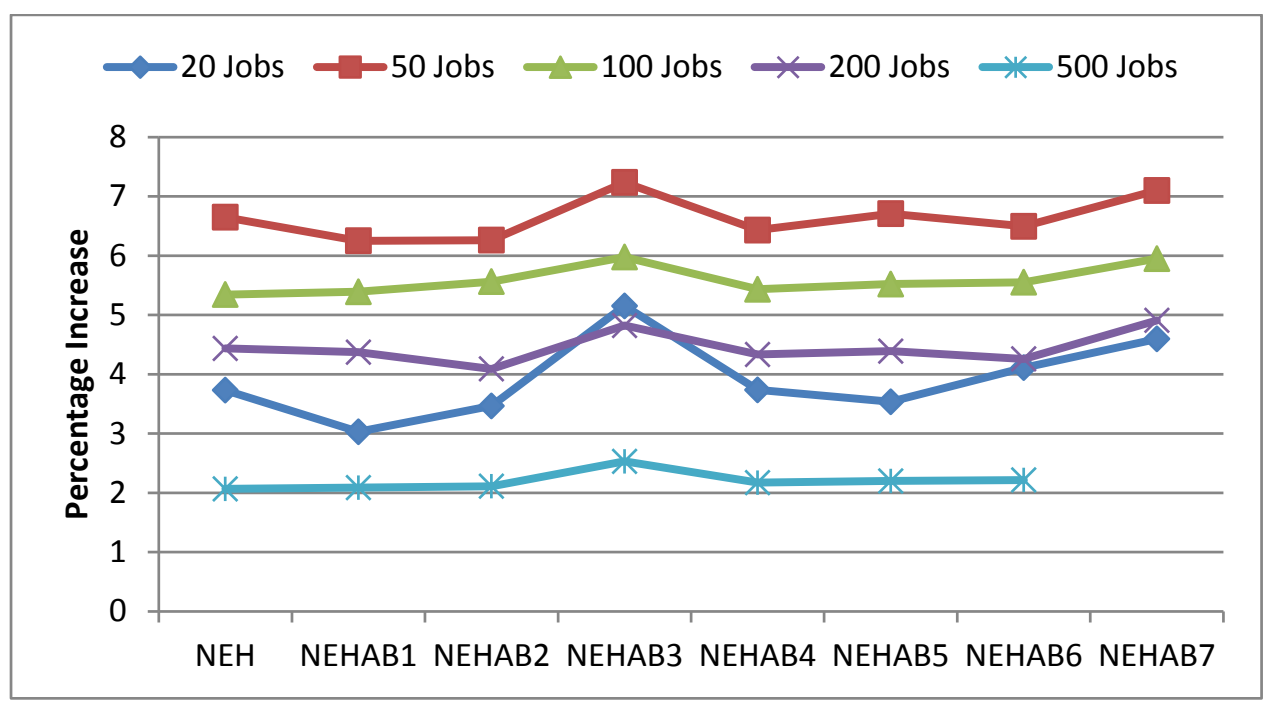

Fig. 3. Percent Increase Plot for 20 Machines 
The algorithms perform better in case of 5 machines, 100 jobs; 10 machines, 200 jobs and 20 machines, 500 jobs problem instances. That is, in the highest number of jobs for a particular number of machines. When the percentage deviations are analyzed, it is more than $5 \%$ in case of 10 machines, 50 jobs; 20 machines, 50 jobs and 20 machines, 100 jobs.

\section{ANOVA}

One way ANOVA has been carried out at 95\% confidence level. The variance analysis has been executed using the MINITAB $17 \circledR$ software.

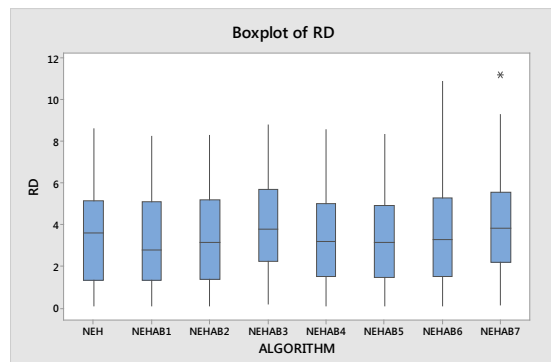

Fig.4. Box Plot for the Relative Deviations

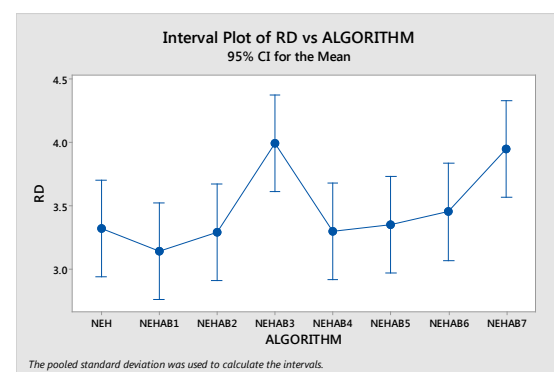

Fig. 5. Interval Plot for the Relative Deviations

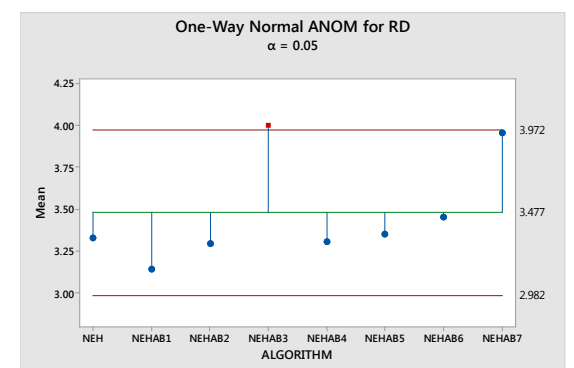

Fig. 6. One Way Normal Analysis of Means for Relative Deviations

The box plot, interval plot and normal analysis of means are shown from Fig. 4 to Fig. 6. The performance of NEHAB1 is better than the others. The means of NEHAB3 and NEHAB7 are above the average level. These two algorithms do not pre-arrange the jobs in any specific order, but, uses the job insertion technique to arrive with the final sequence. However, the performance of these two also better than many heuristics proposed over the years, the deviations being below $4 \%$. This clearly shows the power of the job insertion technique in minimizing the makespan in permutation flow shop problems. The output produced by the software is reproduced below:

Analysis of Variance

$\begin{array}{lrrrrr}\text { Source } & \text { DF } & \text { Adj SS } & \text { Adj MS } & \text { F-Value } & \text { P-Value } \\ \text { ALGORITHM } & 7 & 85.54 & 12.220 & 2.70 & 0.009 \\ \text { Error } & 952 & 4308.99 & 4.526 & & \\ \text { Total } & 959 & 4394.53 & & & \end{array}$

Model Summary

$\begin{array}{rrrr}S & R-s q & R-s q(\operatorname{adj}) & R-s q(\text { pred }) \\ 2.12750 & 1.95 \% & 1.23 \% & 0.29 \%\end{array}$

Means

$\begin{array}{lrrrrr}\text { ALGORITHM } & \text { N } & \text { Mean } & \text { StDev } & 95 \% \text { CI } \\ \text { NEH } & 120 & 3.325 & 2.121 & (2.944,3.706) \\ \text { NEHAB1 } & 120 & 3.143 & 2.037 & (2.762, & 3.524) \\ \text { NEHAB2 } & 120 & 3.291 & 2.117 & (2.910, & 3.672) \\ \text { NEHAB3 } & 120 & 3.997 & 2.209 & (3.616, & 4.378) \\ \text { NEHAB4 } & 120 & 3.303 & 2.052 & (2.922,3.684) \\ \text { NEHAB5 } & 120 & 3.351 & 2.096 & (2.970, & 3.733) \\ \text { NEHAB6 } & 120 & 3.453 & 2.172 & (3.072,3.834) \\ \text { NEHAB7 } & 120 & 3.953 & 2.209 & (3.572, & 4.334)\end{array}$

Pooled StDev $=2.12750$

The null hypothesis and the alternate are:

$\mathrm{H}_{0}$ : all means are same

$\mathrm{H}_{\mathrm{A}}$ : at least one mean is different 
In the analysis, the F value is high (2.70) and the $\mathrm{P}$ value is $<0.05(0.009)$ and hence, the Null Hypothesis is rejected. The algorithms are statistically different.

\section{Interaction and Surface Plots}

For validating further, interaction and Surface plots have been drawn using MINITAB17® software for the results obtained. The maximum order of terms in the model has been set as 2 .

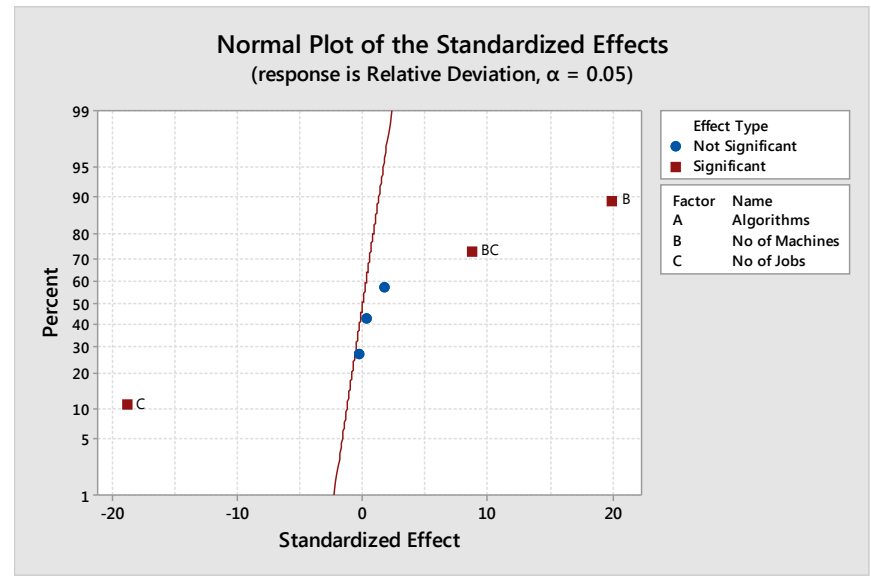

Fig. 7. Normal Plot of the Standardized Effects

In the analysis, algorithm ' 0 ' represents the NEH algorithm, ' 1 ' represents the NEHAB1 algorithm and so on. According to the Normal Plot of the Standardized Effects (Fig. 7), the factors B (number of machines), $\mathrm{C}$ (number of jobs) and $\mathrm{BC}$ have a significant effect on the response (Relative Deviation) for a particular algorithm.

Analysis of Variance

\begin{tabular}{|c|c|c|c|c|c|}
\hline Source & $\mathrm{DF}$ & Adj SS & Adj MS & F-Value & P-Value \\
\hline Model & 7 & 2358.57 & 336.938 & 157.55 & 0.000 \\
\hline 1-Linear & 3 & 882.81 & 294.271 & 137.60 & 0.000 \\
\hline Algorithms & 1 & 5.51 & 5.515 & 2.58 & 0.109 \\
\hline No of Machines & 1 & 852.26 & 852.264 & 398.51 & 0.000 \\
\hline No of Jobs & 1 & 762.00 & 762.00 & 456.30 & 0.000 \\
\hline 2-Way Interactions & 3 & 164.13 & 54.710 & 25.58 & 0.000 \\
\hline Algorithms*No of Machines & 1 & 0.82 & 0.824 & 0.39 & 0.535 \\
\hline Algorithms*No of Jobs & 1 & 0.61 & 0.609 & 0.28 & 0.594 \\
\hline 3-Way Interactions & 1 & 1.34 & 1.344 & 0.63 & 0.428 \\
\hline Algorithms*No of Machines*No of Jobs & 1 & 1.34 & 1.344 & 0.63 & 0.428 \\
\hline Error & 952 & 2035.97 & 2.139 & & \\
\hline Lack-of-Fit & 88 & 1070.95 & 12.170 & 10.90 & 0.000 \\
\hline Pure Error & 864 & 965.02 & 1.117 & & \\
\hline Total & 959 & 4394.53 & & & \\
\hline \multicolumn{6}{|l|}{ Model Summary } \\
\hline S & R-Sq(sdj) & R-sq(pred) & & & \\
\hline
\end{tabular}

Coded Coefficients

\begin{tabular}{|c|c|c|c|c|c|c|}
\hline Term & Effect & Coef & SE Coef & T-Value & P-Value & VIF \\
\hline Constant & & 1.099 & 0.143 & 7.68 & 0.000 & \\
\hline Algorithms & 0.702 & 0.351 & 0.219 & 1.61 & 0.109 & 9.20 \\
\hline No of Machines & 6.096 & 3.048 & 0.153 & 19.96 & 0.000 & 7.33 \\
\hline No of Job2 & -6.686 & -3.343 & 0.177 & -18.88 & 0.000 & 4.14 \\
\hline Algorithms*No of Machines & -0.289 & -0.145 & 0.233 & -0.62 & 0.535 & 7.36 \\
\hline Algorithms*No of Job2 & 0.289 & 0.144 & 0.271 & 0.53 & 0.594 & 9.10 \\
\hline No of Machines*No of Job2 & 3.193 & 1.596 & 0.183 & 8.74 & 0.000 & 6.87 \\
\hline
\end{tabular}


The p-value is small ( $<0.05$, a level of significance) for the factors; the number of machines, the number of jobs and the interaction between the number of machines * number of jobs. They have statistically significant effect on the response. However, the algorithms and the other interactions are significantly different statistically.

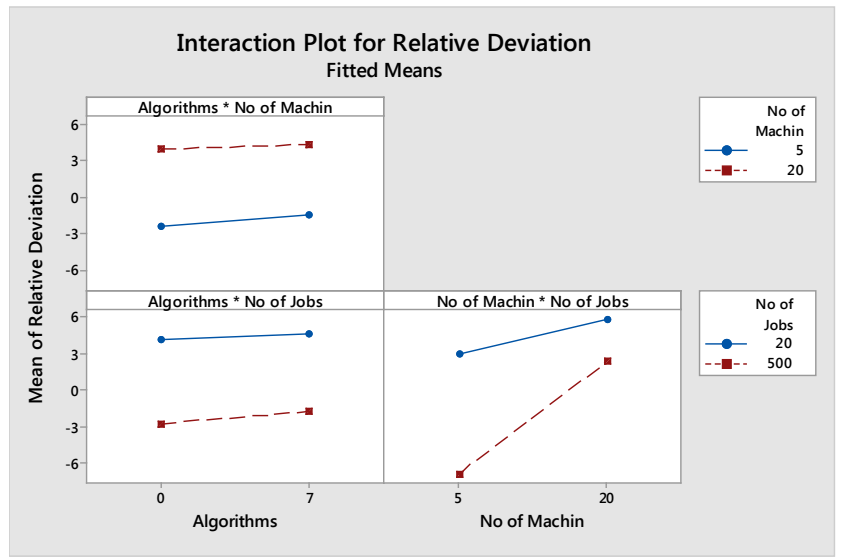

Fig. 8. Interaction Plot for Relative Deviation

The interaction plot is presented in Fig. 8. For a particular algorithm, the relative deviation is less for the combination of less number of machines with a large number of jobs.

The surface plots of relative deviations are shown from Fig. 9 and Fig. 10. The relative deviation is high as the number of machines increases. Algorithm 3 (NEHAB3) reports the highest deviations in general followed by the Algorithm 7 (NEHAB7). The performance of NEHAB1 is better than the others. Similarly, the relative deviation is decreasing with the increase in the number of jobs. This reaffirms the analysis of variance.

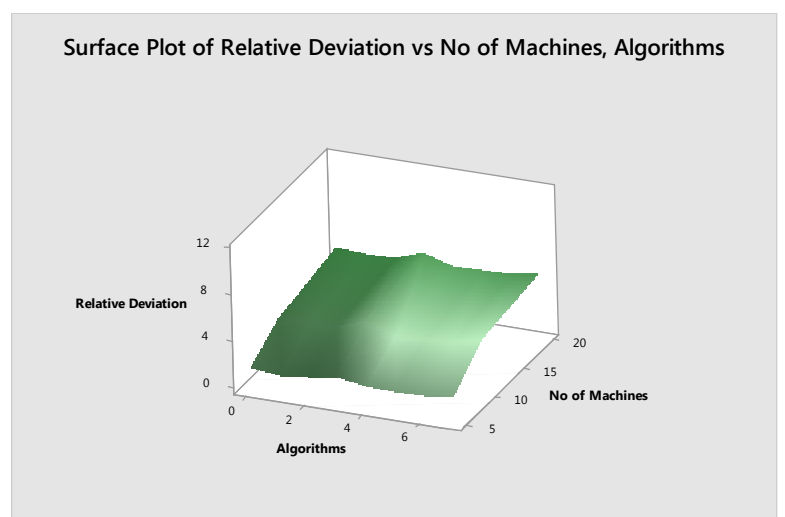

Fig. 9. Surface Plot of Relative Deviation vs No of Machines, Algorithms

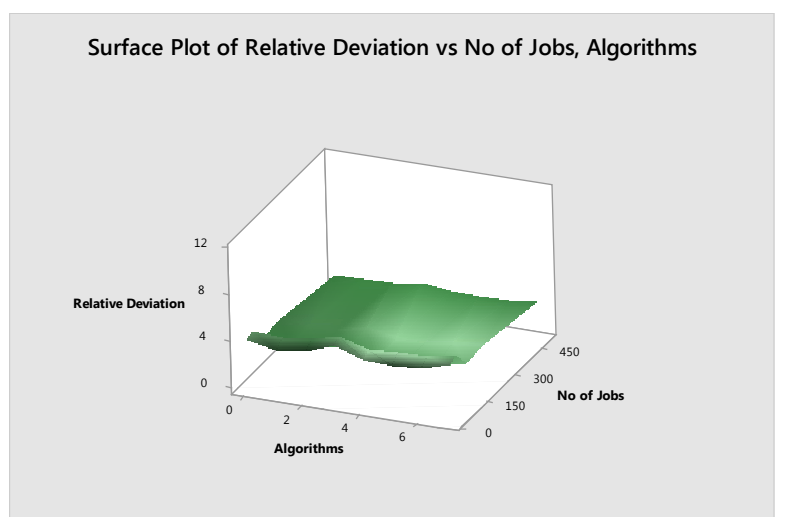

Fig. 10. Surface Plot of Relative Deviation vs No of Jobs, Algorithms

\section{Conclusion and Future Work}

In this paper, 7 heuristics have been analyzed along with the popular NEH heuristic. The variants are obtained by varying the initial partial sequence. Except in two, the jobs are arranged initially in non increasing order of their total processing times. The average percentage increase in the makespan from the known upper bounds is below $4 \%$ for all the cases which is better than many known simple heuristics. Simply changing the initial partial sequence improves the makespan in three algorithms. It is concluded that, in addition to selecting the first two jobs as the initial partial sequence, other partial sequences also yield better results than the original NEH algorithm. The job insertion technique is the most powerful component of the NEH algorithm followed by the initial arrangement of jobs in non increasing order of 
their total processing times. NEH algorithm has been the most popular among the simple heuristics in minimizing the makespan in flow shop scheduling problems. The results obtained using NEH have been used by many meta-heuristics to refine the solution further. Many improvement tools have been proposed for the NEH over the years. Three algorithms among the seven analyzed in this paper are the simpler variants of the $\mathrm{NEH}$ with the same complexity level, but yield better results. These may be analyzed further to compare the performance still better. Further research includes the application of the improvement tools proposed by other researchers for the NEH algorithm in these algorithms also and analyzing the effects for different benchmark problem instances.

\section{References}

Ancău, M. (2012). On solving flowshop scheduling problems. Proceedings of the Romanian Academy. Series A, 13(1), 71-79

Baskar, A., \& Xavior, M. (2013). Optimization of makespan in flow shop scheduling problems using combinational NEH family of heuristics-An analysis. International Journal of Applied Engineering Research, 8(10), 1205-1217.

Campbell, H. G., Dudek, R. A., \& Smith, M. L. (1970). A heuristic algorithm for the $\mathrm{n}$ job, m machine sequencing problem. Management Science, 16(10), B-630.

Caraffa, V., Ianes, S., Bagchi, T. P., \& Sriskandarajah, C. (2001). Minimizing makespan in a blocking flowshop using genetic algorithms. International Journal of Production Economics, 70(2), 101-115.

Chakraborty, U. K., \& Laha, D. (2007). An improved heuristic for permutation flowshop scheduling. International Journal of Information and Communication Technology, 1(1), 89-97.

Chen, C. L., Vempati, V. S., \& Aljaber, N. (1995). An application of genetic algorithms for flow shop problems. European Journal of Operational Research, 80(2), 389-396.

Dannenbring, D. G. (1977). An evaluation of flow shop sequencing heuristics. Management Science, 23(11), 1174-1182.

Dong, X., Huang, H., \& Chen, P. (2008). An improved NEH-based heuristic for the permutation flowshop problem. Computers \& Operations Research, 35(12), 3962-3968.

Erdoğmuş, P. (2010). Particle swarm optimization performance on special linear programming problems. Scientific Research and Essays, 5(12), 1506-1518.

Fernandez-Viagas, V., \& Framinan, J. M. (2014). On insertion tie-breaking rules in heuristics for the permutation flowshop scheduling problem. Computers \& Operations Research, 45, 60-67.

Framinan, J. M., Leisten, R., \& Rajendran, C. (2003). Different initial sequences for the heuristic of Nawaz, Enscore and Ham to minimize makespan, idletime or flowtime in the static permutation flowshop sequencing problem. International Journal of Production Research, 41(1), 121-148.

Gantt, H. L. (1919). Organizing for work. Harcourt, Brace and Howe.

Gajpal, Y., \& Rajendran, C. (2006). An ant-colony optimization algorithm for minimizing the completion-time variance of jobs in flowshops. International Journal of Production Economics, 101(2), 259-272.

Garey, M. R., Johnson, D. S., \& Sethi, R. (1976). The complexity of flowshop and jobshop scheduling. Mathematics of Operations Research, 1(2), 117-129.

Gupta, A., \& Chauhan, S. (2015). A heuristic algorithm for scheduling in a flow shop environment to minimize makespan. International Journal of Industrial Engineering Computations, 6(2), 173-184.

Gupta, J. N. (1971). A functional heuristic algorithm for the flowshop scheduling problem. Operational Research Quarterly, 39-47.

Hundal, T. S., \& Rajgopal, J. (1988). An extension of Palmer's heuristic for the flow shop scheduling problem. The International Journal Of Production Research, 26(6), 1119-1124.

Ishibuchi, H., Misaki, S., \& Tanaka, H. (1995). Modified simulated annealing algorithms for the flow shop sequencing problem. European Journal of Operational Research, 81(2), 388-398.

Johnson, S. M. (1954). Optimal two-and three-stage production schedules with setup times included. Naval Research Logistics Quarterly, 1(1), 61-68. 
Kalczynski, P. J., \& Kamburowski, J. (2007). On the NEH heuristic for minimizing the makespan in permutation flow shops. Omega, 35(1), 53-60.

Kalczynski, P. J., \& Kamburowski, J. (2008). An improved NEH heuristic to minimize makespan in permutation flow shops. Computers \& Operations Research, 35(9), 3001-3008.

Koulamas, C. (1998). A new constructive heuristic for the flowshop scheduling problem. European Journal of Operational Research, 105(1), 66-71.

Land, A. H., \& Doig, A. G. (1960). An automatic method of solving discrete programming problems. Econometrica: Journal of the Econometric Society, 497-520.

Liu, G., Song, S., \& Wu, C. (2012). Two techniques to improve the NEH algorithm for flow-shop scheduling problems. In Advanced Intelligent Computing Theories and Applications. With Aspects of Artificial Intelligence (pp. 41-48). Springer Berlin Heidelberg.

Murata, T., Ishibuchi, H., \& Tanaka, H. (1996). Genetic algorithms for flowshop scheduling problems. Computers \& Industrial Engineering, 30(4), 1061-1071.

Nagano, M. S., \& Moccellin, J. V. (2002). A high quality solution constructive heuristic for flow shop sequencing. Journal of the Operational Research Society, 53(12), 1374-1379.

Nawaz, M., Enscore, E. E., \& Ham, I. (1983). A heuristic algorithm for the m-machine, n-job flow-shop sequencing problem. Omega, 11(1), 91-95.

Nowicki, E., \& Smutnicki, C. (1996). A fast tabu search algorithm for the permutation flow-shop problem. European Journal of Operational Research, 91(1), 160-175.

Ogbu, F. A., \& Smith, D. K. (1990). The application of the simulated annealing algorithm to the solution of the $\mathrm{n} / \mathrm{m} / \mathrm{C}$ max flowshop problem. Computers \& Operations Research, 17(3), 243-253.

Onwubolu, G., \& Davendra, D. (2006). Scheduling flow shops using differential evolution algorithm. European Journal of Operational Research, 171(2), 674-692.

Osman, I. H., \& Potts, C. N. (1989). Simulated annealing for permutation flow-shop scheduling. Omega, 17(6), 551-557.

Palmer, D. (1965). Sequencing jobs through a multi-stage process in the minimum total time--a quick method of obtaining a near optimum. OR, 101-107.

Pinedo, M. L. (2012). Scheduling: Theory, Algorithms, and Systems. Springer Science \& Business Media.

Ponnambalam, S. G., Aravindan, P., \& Chandrasekaran, S. (2001). Constructive and improvement flow shop scheduling heuristics: an extensive evaluation. Production Planning \& Control, 12(4), 335-344.

Pour, H. D. (2001). A new heuristic for the n-job, m-machine flow-shop problem. Production Planning \& Control, 12(7), 648-653.

Rajendran, C. (1993). Heuristic algorithm for scheduling in a flowshop to minimize total flowtime. International Journal of Production Economics, 29(1), 65-73.

Reza Hejazi*, S., \& Saghafian, S. (2005). Flowshop-scheduling problems with makespan criterion: a review. International Journal of Production Research, 43(14), 2895-2929.

Ribas, I., \& Mateo, M. (2010). Improvement tools for NEH based heuristics on permutation and blocking flow shop scheduling problems. In Advances in Production Management Systems. New Challenges, New Approaches (pp. 33-40). Springer Berlin Heidelberg.

Reeves, C. R. (1995). A genetic algorithm for flowshop sequencing. Computers \& Operations Research, 22(1), 5-13.

Ronconi, D. P. (2004). A note on constructive heuristics for the flowshop problem with blocking. International Journal of Production Economics, 87(1), 39-48.

Ruiz, R., \& Maroto, C. (2005). A comprehensive review and evaluation of permutation flowshop heuristics. European Journal of Operational Research,165(2), 479-494.

Sarin, S., \& Lefoka, M. (1993). Scheduling heuristic for the n-jobm-machine flow shop. Omega, 21(2), 229-234.

Sayadi, M., Ramezanian, R., \& Ghaffari-Nasab, N. (2010). A discrete firefly meta-heuristic with local search for makespan minimization in permutation flow shop scheduling problems. International Journal of Industrial Engineering Computations, 1(1), 1-10. 
Semančo, P., \& Modrák, V. (2012). A Comparison of Constructive Heuristics with the Objective of Minimizing Makespan in the Flow-Shop Scheduling Problem. Acta Polytechnica Hungarica, 9(5).

Singhal, E., Singh, S., \& Dayma, A. (2012). An Improved Heuristic for Permutation Flow Shop Scheduling. In NEH ALGORITHM). International Journal of Computational Engineering Research, 2(6), 95-100.

Suliman, S. M. A. (2000). A two-phase heuristic approach to the permutation flow-shop scheduling problem. International Journal of Production Economics,64(1), 143-152.

Taillard, E. (1990). Some efficient heuristic methods for the flow shop sequencing problem. European journal of Operational research, 47(1), 65-74.

Taillard, E. (1993). Benchmarks for basic scheduling problems. European Journal of Operational Research, 64(2), 278-285.

Widmer, M., \& Hertz, A. (1989). A new heuristic method for the flow shop sequencing problem. European Journal of Operational Research, 41(2), 186-193.

Xiao-ping, L., Yue-Xuan, W., \& Cheng, W. (2004, June). Heuristic algorithms for large flowshop scheduling problems. In Intelligent Control and Automation, 2004. WCICA 2004. Fifth World Congress on (Vol. 4, pp. 2999-3003). IEEE.

Yin, M., Zou, T., \& Gu, W. (2010). Reverse Bridge Theorem under Constraint Partition. Mathematical Problems in Engineering, 2010. 